\title{
Jens Thiel zum Professor für Rheumatologie berufen
}

Im November 2021 wurde Prof. Dr. Jens Thiel für das Fach „Rheumatologie“ an die Medizinische Universität Graz berufen.

Quelle: Presseaussendung der Medizinischen Universität Graz

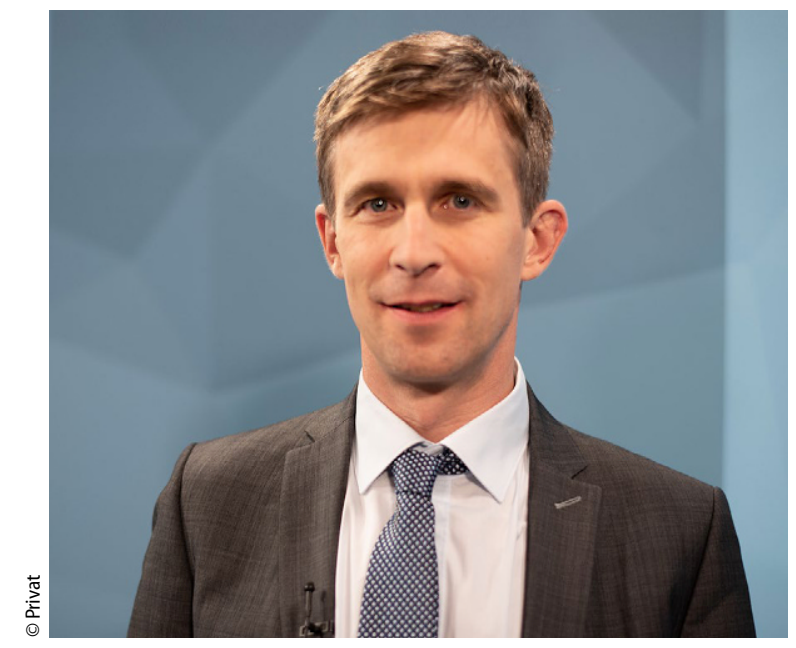

$\Delta$ Prof. Dr. Jens Thiel dort die Stelle des leitenden Oberarztes und Stellvertreters des ärztlichen Direktors inne.

\section{Fest verankert in Forschung und Lehre}

in Rotenburg/Fulda, Deutschland, geboren und studierte Humanmedizin an der Albert-Ludwigs-Universität Freiburg, wo er 2002 promovierte. Nach Abschluss des Studiums begann er mit seiner Ausbildung zum Facharzt für Innere Medizin an der Klinik für Rheumatologie und Klinische Immunologie am Universitätsklinikum Freiburg. Dort erhielt er die Facharztanerkennung für Innere Medizin und Rheumatologie.

2015 habilitierte sich Thiel an der Albert Ludwigs-Universität Freiburg im Fach „Innere Medizin“. Ab 2016 bis zu seiner Berufung an die Medizinische Universität Graz leitete Jens Thiel die Sektion für Immunvaskulitiden an der Klinik für Rheumatologie und Klinische Immunologie am Universitätsklinikum Freiburg. Zuletzt hatte er

Den Schwerpunkt seines wissenschaftlichen Interesses bildet die Erforschung von Störungen der immunologischen Gedächtnisbildung, welche zu Autoimmunität und Inflammation sowie zu Immunschwäche führen können. Dabei stehen translationale Fragestellungen, die aus klinischen Beobachtungen entstehen und deren Beantwortung unmittelbar zurVerbesserung der Patient*innenversorgung führt, im Vordergrund. Thiel ist insbesondere an der Entstehung entzündlicher Gelenkserkrankungen wie der rheumatoiden Arthritis und der Krankheitsentwicklung immunologisch-vermittelter Gefäßerkrankungen interessiert. Zur Erforschung dieser Erkrankungen steht inm die Biobank der MedUni Graz zur Verfügung.

Darüber hinaus erlauben die an der MedUni Graz bereits vorhanden Forschungsschwerpunkte im Bereich Inflammation und Metabolismus, sowie die Ausstattung des Zentrums für Medizinische Forschung, Kooperationen an den Schnittstellen von Immunologie und, unter anderem, Inflammation und Metabolismus mit einer Vielzahl anderer Fachdisziplinen wie z.B. der (kardio)-vaskulären Medizin.

Ein weiterer Schwerpunkt von Thiel wird in der Übertragung grundlagenwissenschaftlicher Erkenntnisse in klinische Studien liegen. Thiel leitet internationale Studien zur Therapie entzündlicher Gefäßerkrankungen. Das wissenschaftliche Profil von Thiel schlägt sich in einer Vielzahl von Publikationen in renommierten Journalen nieder. Zudem ist er Gutachter mehrerer international bedeutender Journals im Bereich der Rheumatologie und Träger hochrangiger Auszeichnungen.

Thiel ist seit vielen Jahren in der Ausbildung von Mediziner*innen engagiert. Der Schwerpunkt seiner Lehrtätigkeit umfasst das Fachgebiet der Rheumatologie und klinischen Immunologie, für die er in grundlagenwissenschaftlichen und praktisch klinischen Lehrveranstaltungen die Begeisterung der Studierenden zu wecken weiß.

Ein besonderes Anliegen ist es Thiel, die rheumatologische Patient ${ }^{*}$ innenversorgung in der Steiermark in Kooperation mit anderen Fachdisziplinen und den niedergelassenen Kolleg*innen, weiter zu verbessern. Dazu plant er unter anderem einen niederschwelligeren Zugang zur universitären Rheumatologie für zuweisende Kolleg*innen mit rheumatologisch-immunologischen Fragestellungen, ebenso wie regelmäBige Patient*innenveranstaltungen zu etablieren beziehungsweise weiter auszubauen.

Hinweis des Verlags. Der Verlag bleibt in Hinblick auf geografische Zuordnungen und Gebietsbezeichnungen in veröffentlichten Karten und Institutsadressen neutral.

rheuma plus $2022 \cdot 21: 12$

https://doi.org/10.1007/s12688-02200494-7

(c) The Author(s), under exclusive licence to Springer-Verlag GmbH Austria, ein Teil von Springer Nature 2022 